\title{
Physicians' perceptions of asymptomatic hyperuricemia in patients with chronic kidney disease: A questionnaire survey
}

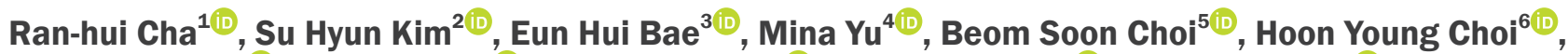

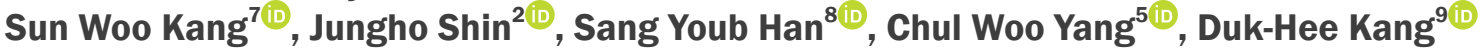 \\ ${ }^{1}$ Department of Internal Medicine, National Medical Center, Seoul, Korea \\ 'Department of Internal Medicine, Chung-Ang University Hospital, Chung-Ang University College of Medicine, Seoul, Korea \\ ${ }^{3}$ Department of Internal Medicine, Chonnam National University Medical School, Gwangju, Korea \\ ${ }^{4}$ Department of Internal Medicine, Seonam Hospital, Seoul, Korea \\ ${ }^{5}$ Department of Internal Medicine, College of Medicine, The Catholic University of Korea, Seoul, Korea \\ ${ }^{6}$ Department of Internal Medicine, Yonsei University College of Medicine, Seoul, Korea \\ ${ }^{7}$ Department of Nephrology, Inje University Busan Paik Hospital, Busan, Korea \\ ${ }^{8}$ Department of Internal Medicine, Ilsan Paik Hospital, Inje University College of Medicine, Goyang, Korea \\ ${ }^{9}$ Department of Internal Medicine, School of Medicine, Ewha Womans University, Seoul, Korea
}

\begin{abstract}
Background: Hyperuricemia is associated with the development and progression of chronic kidney disease (CKD) as well as cardiovascular diseases. However, there is no consistent recommendation regarding the treatment of asymptomatic hyperuricemia (AHU) in CKD patients. Here, we surveyed Korean physicians' perceptions regarding the diagnosis and management of AHU in CKD patients.

Methods: Questionnaires on the management of AHU in CKD patients were emailed to regular members registered with the Korean Society of Nephrology.

Results: A total of 158 members answered the questionnaire. Among the respondents, 49.4\%/41.1\% were considered hyperuricemic in male CKD patients whereas 36.7\%/20.9\% were considered hyperuricemic in female CKD patients when defined by serum uric acid level over $7.0 / 8.0 \mathrm{mg} / \mathrm{dL}$, respectively. A total of $80.4 \%$ reported treating AHU in CKD patients. The most important reasons to treat AHU in CKD patients were renal function preservation followed by cerebro-cardiac protection. Majority of respondents (59.5\%) thought that uric acid-lowering agents (ULAs) were the most effective method for controlling serum uric acid levels. Approximately $80 \%$ chose febuxostat as the preferred medication. A total of $32.3 \%$ and $31.0 \%$, respectively, initiated ULA treatment if the serum
\end{abstract}

Received January 13, 2019; Revised March 15, 2019; Accepted March 18, 2019

Edited by Dong-Ryeol Ryu, Ewha Womans University, Seoul, Korea

Correspondence: Duk-Hee Kang

Division of Nephrology, Department of Internal Medicine, Ewha Womans University Seoul Hospital, 26o Gonghang-daero, Gangseo-gu, Seoul 07804, Korea. E-mail: dhkang@ewha.ac.kr

Ran-hui Cha

Division of Nephrology, Department of Internal Medicine, National Medical Center, 245 Eulji-ro, Jung-gu, Seoul o4564, Korea. E-mail:reginaprayer@gmail.com

Copyright (c) 2019 by The Korean Society of Nephrology

(a) This is an open-access article distributed under the terms of the Creative Commons Attribution Non-Commercial License (http://creativecommons.org/ licenses/by-nc-nd/4.0/), which permits unrestricted non-commercial use, distribution, and reproduction in any medium, provided the original work is properly cited. 
uric acid level was more than 8.0 or $9.0 \mathrm{mg} / \mathrm{dL}$, respectively. In addition, $39.2 \%$ and $30.4 \%$ answered that target serum uric acid levels of less than 6.0 or $7.0 \mathrm{mg} / \mathrm{dL}$, respectively, were appropriate. The two major hurdles to prescribing ULAs were concerns of adverse reactions and the existing lack of evidence (i.e., the absence of Korean guidelines).

Conclusion: Most Korean physicians treat AHU in CKD patients to prevent CKD progression and cerebrocardiovascular complications.

Keywords: Asymptomatic hyperuricemia, Chronic kidney disease, Survey, Treatment

\section{Introduction}

Hyperuricemia is a very common biochemical aberration and the prevalence is increasing due to changes in diet, an aging population, and earlier screenings [1]. Uric acid comes from both internal (muscle, liver, intestine) and external (diet) sources, with two-thirds excreted through the kidneys [2]. Approximately $20 \%$ to $25 \%$ of adult men experience hyperuricemia, and this prevalence increases up to more than $60 \%$ in advanced chronic kidney disease (CKD) patients because of their decreased urinary uric acid excretion [1]. Two-thirds of hyperuricemia cases remain asymptomatic. Asymptomatic hyperuricemia (AHU) presents as elevated serum uric acid levels without symptoms or signs of monosodium urate crystal deposition disease, hypertension, CKD, cardiovascular disease, or insulin resistance syndrome.

Although the incidence of hyperuricemia and gout in Korean adults is also increasing, epidemiologic studies remain limited. The prevalence of hyperuricemia ( $>7.0$ $\mathrm{mg} / \mathrm{dL}$ ) was $4.6 \%$ in a hospital medical check-up cohort in 2003 [3]. A cohort of 10,802 hyperuricemia-free men aged 30 to 59 years who underwent annual or biennial medical check-ups in a hospital from 2002 to 2009 revealed an incidence rate of hyperuricemia $(>7.0 \mathrm{mg} / \mathrm{dL})$ of 48.7 per 1,000 person-years (95\% confidence interval, 46.8-80.7) [4]. More recently, a report on the prevalence of hyperuricemia using data from the 2016 Korean National Health and Nutrition Examination Survey was released, where the age-standardized prevalence of hyperuricemia in the general Korean population was $11.4 \%$ ( $17.0 \%$ in men and $5.9 \%$ in women) [5]. Unfortunately, however, there was no evidence of the incidence or prevalence of hyperuricemia in Korean CKD patients.

Hyperuricemia is thought to be associated with decreased renal function through increased oxidative stress, increased endothelial dysfunction, systemic glomerular hypertension, and reduced renal blood flow [6]. Decreased renal function aggravates uric acid excretion impairment in the kidneys and is cyclical in nature. Several studies have reported that hyperuricemia is associated with the development and progression of CKD as well as cardio-metabolic conditions [7-10]. Recently, the Chronic Renal Insufficiency Cohort (CRIC) study revealed that a relationship between baseline serum uric acid levels and progression to end-stage renal disease (ESRD) requiring dialysis and transplantation [11]. Higher baseline serum uric acid levels were associated with more renal outcomes, and the relationship was stronger in patients with an estimated glomerular filtration rate (eGFR) of 45 $\mathrm{mL} / \mathrm{min} / 1.73 \mathrm{~m}^{2}$ or more.

We hypothesize that lowering the serum uric acid level could delay renal disease progression and reduce cardiovascular complications. In addition, an effective way to control serum uric acid levels is the prescription of uric acid-lowering agents (ULAs) such as allopurinol, febuxostat, and uricosuric agents.

The Japanese Society of Gout and Nucleic Acid Metabolism recommends treating AHU when either serum uric acid level is greater than $9 \mathrm{mg} / \mathrm{dL}$ or when serum uric acid is greater than $8 \mathrm{mg} / \mathrm{dL}$ and is accompanied by renal damage, urinary lithiasis, hypertension, ischemic heart disease, diabetes, or metabolic syndrome [12]. However, uric acid-lowering therapy for AHU is not recommended for use in the United States (US) and Europe because of conflicting results [13]. Separately, the Korean Rheumatology Society recommends a combination of guidelines for the management of AHU [14]; specifically, they suggest treating AHU if the serum uric acid level is greater than $9 \mathrm{mg} / \mathrm{dL}$ and to search for causes and lifestyle modifications at six months prior to treatment if the serum uric acid level is between 7.0 and $9.0 \mathrm{mg} / \mathrm{dL}$. However, 
there are no specific Korean guidelines for the management of AHU in CKD patients.

The practice pattern of the management of AHU in CKD patients has not yet been surveyed. This study aimed to clarify how Korean nephrologists manage AHU in CKD patients.

\section{Methods}

\section{Study design}

We performed a questionnaire survey to determine physicians' perceptions of AHU in CKD patients from July 9, 2018 to August 6, 2018. We sent and received emails to and from regular members registered with the Korean Society of Nephrology (KSN). The questionnaire was designed as follows: 1) definition of hyperuricemia in CKD patients $(>6.0,>7.0,>8.0,>9.0$, and $>10.0 \mathrm{mg} / \mathrm{dL}$ ); 2) necessities and effective methods of lowering serum uric acid levels (yes vs. no); 3 ) the purpose of managing AHU (gout prevention, nephrolithiasis prevention, renal preservation, and cerebro-cardiovascular protection); 4) evaluation of the urinary uric acid excretion (yes vs. no); 5) the initial ( $>6.0,>7.0,>8.0,>9.0,>10.0 \mathrm{mg} / \mathrm{dL}$ ) and the target $(<4.0,<5.0,<6.0,<7.0,<8.0 \mathrm{mg} / \mathrm{dL})$ serum uric acid levels for prescribing ULAs; 6) preferred medication (allopurinol vs. febuxostat vs. benzbromarone vs. others); 7) evaluation of the HLA-B58:01 allele before prescribing allopurinol (yes vs. no); 8) prescribing doses of allopurinol and febuxostat (open question about the initial and the maximum doses); and 9) hurdles to prescribing ULAs (multiple-choice format [choices of two out of seven], including nonpharmacologic intervention first, too many concomitant medications, fear of adverse reactions, unclear treatment effects, the lack of evidence [i.e., the absence of Korean guidelines], patients' refusal, not fitting insurance criteria).

This study was approved and got a waiver of informed consent by the Institutional Review Board of the National Medical Center in Seoul, Korea (H-1811-096-001).

\section{Statistical analysis}

Descriptive statistics were used to summarize survey responses. The chi-square test was used to compare categorical variables between groups. All statistical analyses were performed with the IBM SPSS Statistics version 20.0 software program (IBM Corp., Armonk, NY, USA). A $P$ value less than 0.05 was considered to be statistically significant.

\section{Results}

We sent email communications to a total of 1,258 regular members who were registered with the KSN and received answers back from 158 (12.6\%) of them.

The mean (median) age of the study participants was 45 (43) years old, and 100 (63.3\%) of the participants were men. Most of the participants were affiliated with referral hospitals (secondary hospitals [34.8\%], tertiary or university-associated hospitals [48.7\%]). The baseline characteristics of the participants are summarized in Table 1.

\section{Definition of hyperuricemia in CKD patients}

Among the respondents, $3.2 \%, 49.4 \%, 36.8 \%, 8.9 \%$, and $1.9 \%$ were considered hyperuricemic in male CKD patients to be greater than 6.0, 7.0, 8.0, 9.0, and $10.0 \mathrm{mg} /$ $\mathrm{dL}$, respectively, while $27.2 \%, 41.1 \%, 20.9 \%, 8.0 \%$, and $1.3 \%$ were considered hyperuricemic in female CKD patients to be greater than $6.0,7.0,8.0,9.0$, and $10.0 \mathrm{mg} /$ dL (Fig. 1). Ninety-three (58.9\%) respondents followed the same criteria for hyperuricemia in both male and fe-

Table 1. Baseline characteristics of survey participants

\begin{tabular}{lc}
\hline \multicolumn{1}{c}{ Variable } & Data $(\mathrm{n}=158)$ \\
\hline Age $(\mathrm{yr})$ & $45 \pm 9(31-70)$ \\
$<40$ & $54(34.2)$ \\
$40-49$ & $56(35.4)$ \\
$50-59$ & $39(24.7)$ \\
$\geq 60$ & $7(4.4)$ \\
Hospital & \\
Primary & $26(16.5)$ \\
Secondary & $55(34.8)$ \\
$\geq$ Tertiary & $77(48.7)$ \\
Medical license issuance year ${ }^{\mathrm{a}}$ & \\
$<1990$ & $9(5.7)$ \\
1990-1999 & $36(22.8)$ \\
$2000-2009$ & $56(35.4)$ \\
$\geq 2010$ & $55(34.8)$ \\
\hline
\end{tabular}

Data are presented as mean \pm standard deviation and median (range) for continuous variables or number (\%) for categorical variables.

a1978-2018. 
male CKD patients $(>6.0 \mathrm{mg} / \mathrm{dL}$, five respondents; $>7.0$ $\mathrm{mg} / \mathrm{dL}, 44$ respondents; $>8.0 \mathrm{mg} / \mathrm{dL}, 30$ respondents; $>9.0 \mathrm{mg} / \mathrm{dL}, 11$ respondents; $>10 \mathrm{mg} / \mathrm{dL}$, three respondents, respectively). Fifty-eight (36.7\%) and four (2.5\%) respondents chose 1 and $2 \mathrm{mg} / \mathrm{dL}$ higher serum uric acid levels in male CKD patients than in female CKD patients, respectively. There was no significant difference in the definition of hyperuricemia among male and female CKD patients according to the hospital hierarchy (linearby-linear association $P=0.1$ and 0.6 for male and female CKD patients, respectively) (Fig. 2).

\section{Intervention for AHU in CKD patients}

A majority of respondents (80.4\%) reported treating AHU in CKD patients. There was no significant difference

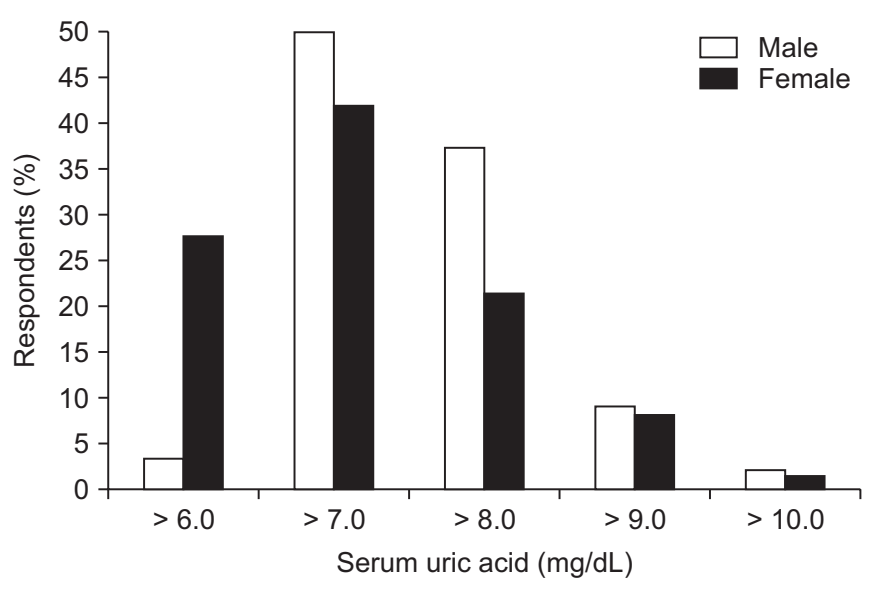

Figure 1. Definition of hyperuricemia in chronic kidney disease patients.

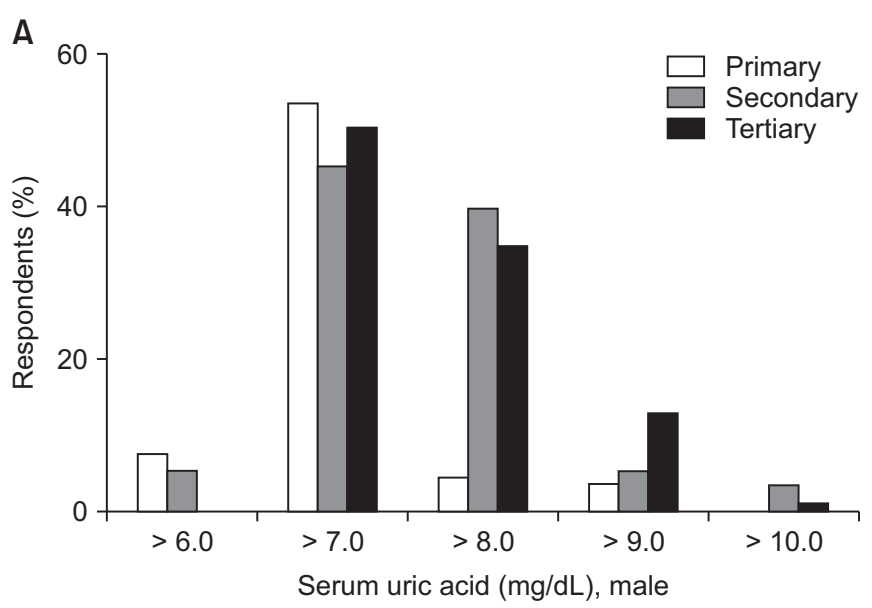

in treating AHU according to the hospital hierarchy (88.5 vs. 76.4 vs. $80.5 \%$ ) (linear-by-linear association $P=0.7$ ).

When we separated the condition according to eGFR, $7.6 \%, 8.2 \%$, and $18.4 \%$ of respondents disagreed about treating AHU in patients with eGFR values of less than 30 , 30 to 60 , and $60 \mathrm{~mL} / \mathrm{min} / 1.73 \mathrm{~m}^{2}$ or more, respectively. ULAs were thought to be the most effective method of controlling serum uric acid levels (59.5\%), followed by lifestyle modification (31.0\%) and a change in prescription to alternative medicines (9.5\%). The most important reasons for treating AHU in CKD patients were renal function preservation followed by cerebro-cardiovascular protection (Table 2).

\section{Evaluation of hyperuricemia}

Only $15.2 \%$ evaluated urinary uric acid excretion by using 24-hour urine uric acid, fractional excretion of

Table 2. Important reasons to treat asymptomatic hyperuricemia in CKD patients

\begin{tabular}{|c|c|c|c|c|}
\hline \multirow{2}{*}{ Variable } & \multicolumn{3}{|c|}{ GFR (mL/min/1.73 m²) } & \multirow{2}{*}{$P$ value } \\
\hline & $<30$ & $30-60$ & $>60$ & \\
\hline No treatment & $12(7.6)$ & $13(8.2)$ & $29(18.4)$ & 0.034 \\
\hline Gout prevention & $26(16.5)$ & $21(13.3)$ & $40(25.3)$ & 0.145 \\
\hline Nephrolithiasis prevention & $2(1.3)$ & $1(0.6)$ & $4(2.5)$ & 0.415 \\
\hline Renal preservation & $81(51.3)$ & $98(62.0)$ & $45(28.5)$ & 0.002 \\
\hline $\begin{array}{l}\text { Cerebro-cardiovascular } \\
\text { protection }\end{array}$ & $35(22.2)$ & $22(13.9)$ & $39(24.7)$ & 0.661 \\
\hline
\end{tabular}

CKD, chronic kidney disease; GFR, glomerular filtration rate.

aLinear-by linear.

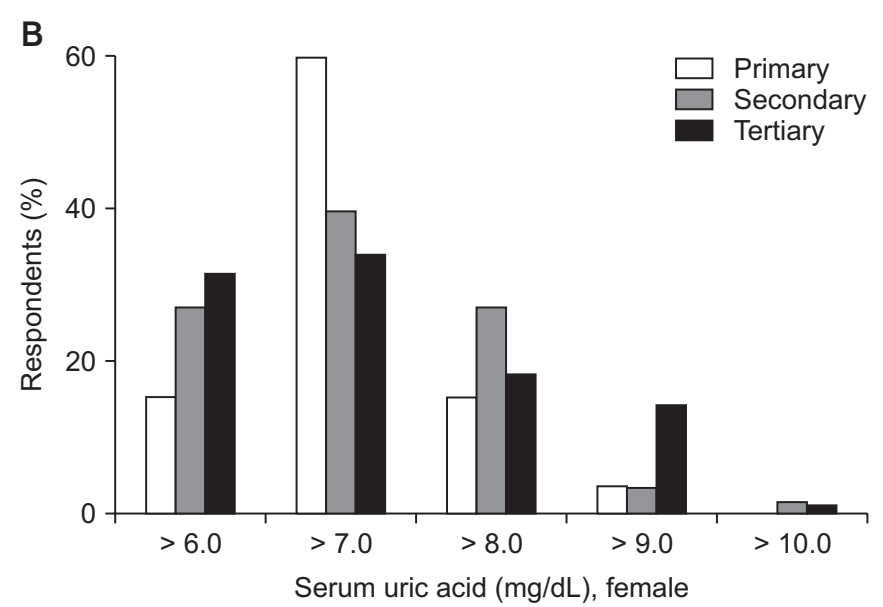

Figure 2. Definition of hyperuricemia in male (A) and female (B) chronic kidney disease patients according to the hospital hierarchy. 

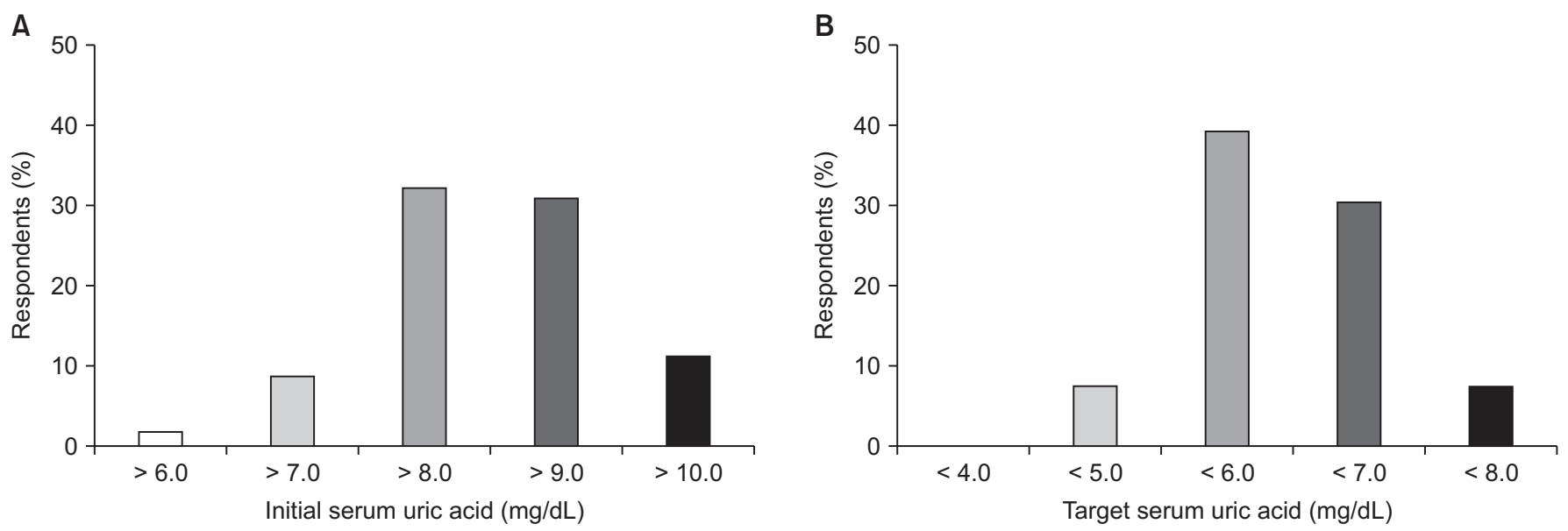

Figure 3. The initial (A) and the target (B) level of serum uric acid in prescribing uric acid-lowering agents.

uric acid from random urine, and urinary stone evaluation through abdominal sonography. In addition, only 4.4\% performed the HLA-B*58:01 allele test, which is the marker for allopurinol-induced severe cutaneous adverse reactions such as Stevens-Johnson syndrome and toxic epidermal necrolysis.

\section{Prescription of uric acid-lowering agents}

Regarding ULA type, $78.1 \%, 19.7 \%$, and $2.2 \%$ of respondents preferred febuxostat, allopurinol, and benzbromarone, respectively. A total of $32.3 \%$ and $31.0 \%$ of the respondents answered they start the prescription of ULAs when serum uric acid level was greater than 8.0 or 9.0 $\mathrm{mg} / \mathrm{dL}$, respectively. Additionally, $39.2 \%$ and $30.4 \%$ of participants targeted serum uric acid levels of less than 6.0 or $7.0 \mathrm{mg} / \mathrm{dL}$, respectively (Fig. 3; Supplementary Table 1 and 2, available online).

Hurdles to prescribing ULAs were as follows: 1) use of a nonpharmacologic intervention first $(9.7 \%), 2)$ too many concomitant medications $(0.6 \%), 3)$ fear of adverse reactions $(36.1 \%), 4)$ unclear treatment effects $(11.0 \%), 5)$ lack of evidence (i.e., the absence of Korean guidelines) $(36.5 \%), 6)$ refusal by patients $(5.5 \%)$, and 7$)$ Not fitting insurance criteria ( $0.6 \%$ ) (Fig. 4$)$. The initial and maximum dosages of allopurinol (Fig. 5) and febuxostat (Fig. 6) were higher in patients with an eGFR value of $30 \mathrm{~mL} /$ $\min / 1.73 \mathrm{~m}^{2}$ or more than in patients with an eGFR of less than $30 \mathrm{~mL} / \mathrm{min} / 1.73 \mathrm{~m}^{2}$ (all $P$ values $<0.001$ ).

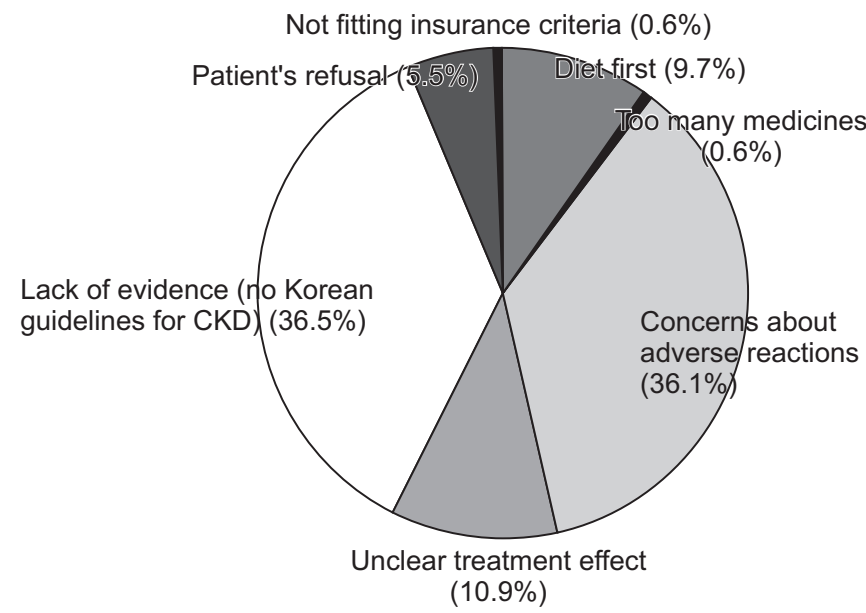

Figure 4. Hurdles to prescribing uric acid-lowering agents. CKD, chronic kidney disease.

\section{Discussion}

To our knowledge, this study is the first survey concerning the management of AHU in CKD patients in Korea. However, the low response rate to the survey reflects the idea that hyperuricemia in CKD patients is not a priority subject even within the KSN.

About half of the responders considered hyperuricemia in CKD patients to be greater than $7.0 \mathrm{mg} / \mathrm{dL}$ of serum uric acid, although approximately one-third chose greater than $8.0 \mathrm{mg} / \mathrm{dL}$ of serum uric acid, regardless of sex, and another one-third chose greater than $6.0 \mathrm{mg} /$ $\mathrm{dL}$ of serum uric acid in female CKD patients. Most respondents (80.4\%) attending CKD patients replied that uric acid-lowering therapy for AHU in CKD patients is 

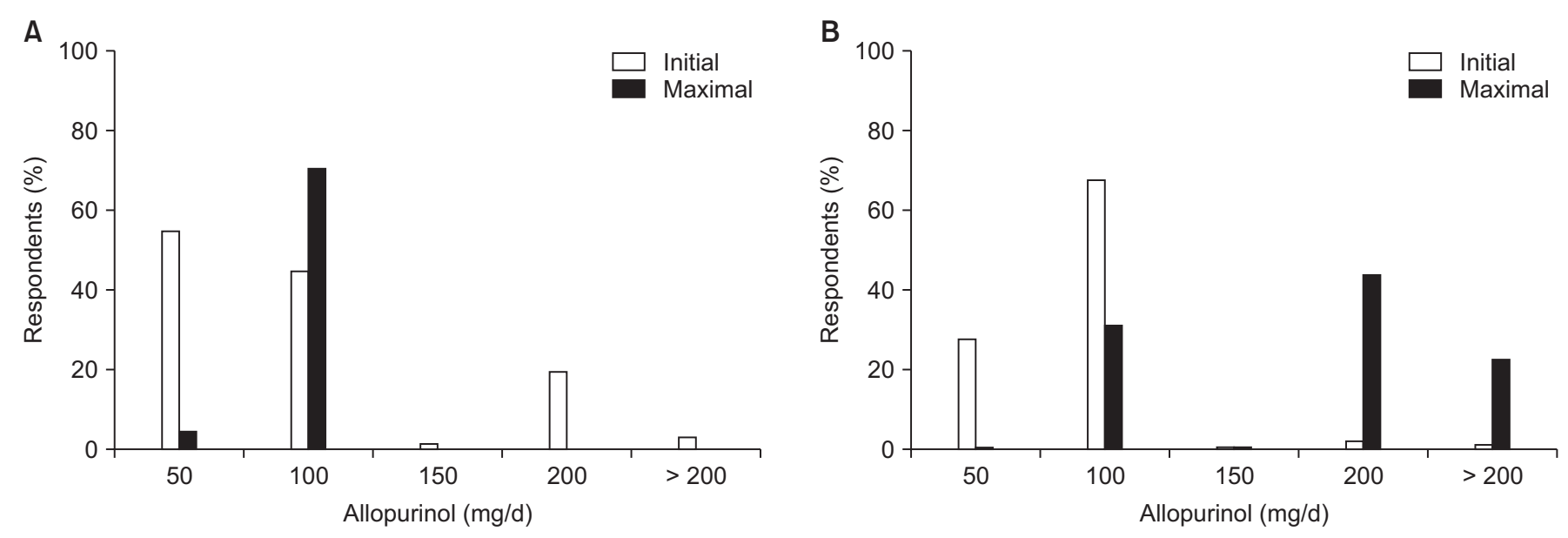

Figure 5. Initial and maximum dosages of allopurinol used to treat asymptomatic hyperuricemia according to renal function. (A) eGFR $<30 \mathrm{~mL} / \mathrm{min} / 1.73 \mathrm{~m}^{2}$; (B) eGFR $\geq 30 \mathrm{~mL} / \mathrm{min} / 1.73 \mathrm{~m}^{2}$.

eGFR, estimated glomerular filtration rate.
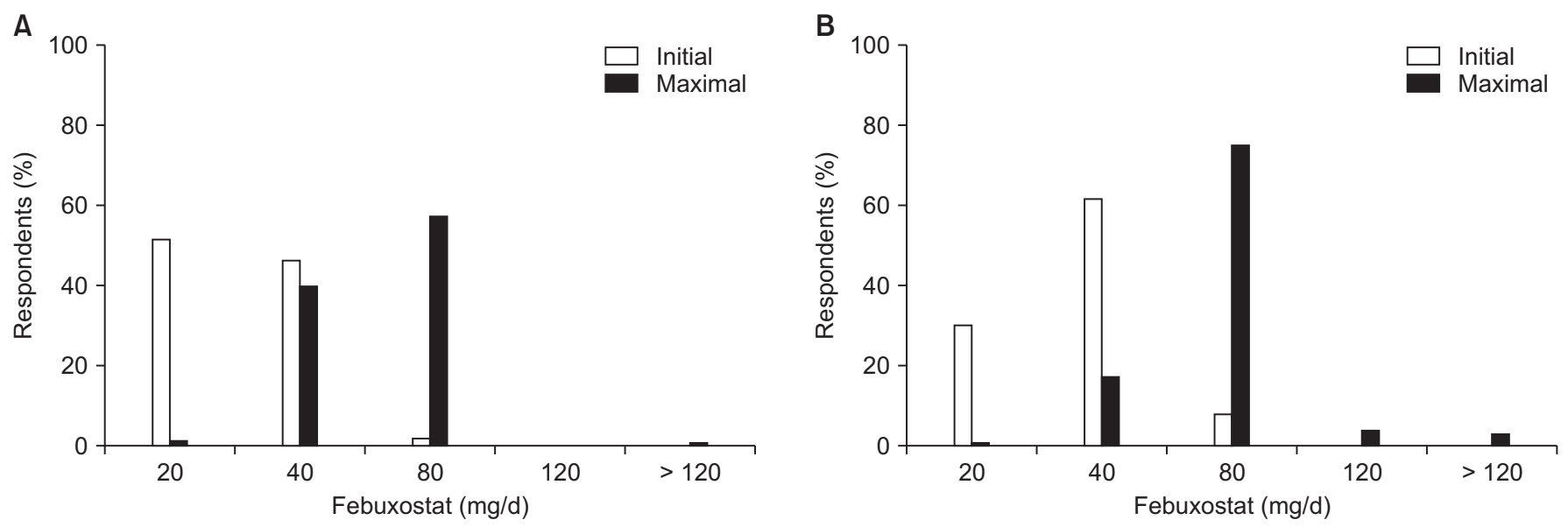

Figure 6. Initial and maximum dosages of febuxostat used to treat asymptomatic hyperuricemia according to renal function. (A) eGFR $<30 \mathrm{~mL} / \mathrm{min} / 1.73 \mathrm{~m}^{2}$; (B) eGFR $\geq 30 \mathrm{~mL} / \mathrm{min} / 1.73 \mathrm{~m}^{2}$.

eGFR, estimated glomerular filtration rate.

necessary. Approximately, $84-89 \%$ and $63 \%$ of Japanese nephrologist agreed to treat AHU in patients with stages 3 to 5 CKD and stage 5D CKD patients, respectively, while only $4 \%$ of rheumatologists in the US prescribed ULAs to patients with AHU $[15,16]$. The most important reasons for treating AHU in CKD patients included the prevention of CKD progression or cerebro-cardiovascular complications. A very small proportion of respondents evaluated urinary uric acid excretion and the HLA-B*58:01 allele. A majority of participants selected febuxostat as the preferred ULA treatment.

About two-thirds of respondents stated that they prescribed ULAs if the serum uric acid level was greater than
$8.0 \mathrm{mg} / \mathrm{dL}$ and targeted a serum uric acid level of less than $7.0 \mathrm{mg} / \mathrm{dL}(<6.0 \mathrm{mg} / \mathrm{dL}, 39.2 \%$ and $<7.0 \mathrm{mg} / \mathrm{dL}$, $30.4 \%)$. Nakaya et al [15] evaluated the initial and target levels of serum uric acid according to CKD stage (CKD stages $3-5$ and $5 \mathrm{D}$ ) and the purpose of treatment (e.g., prevention of gout, urolithiasis, CKD, cardiovascular events [CVEs]). The target levels were different according to every CKD stage and the purpose of treatment. Generally, the initial level of serum uric acid to treat AHU increased as renal function decreased (from 8.1 to $9.5 \mathrm{mg}$ / $\mathrm{dL}$ ). However, the target level of serum uric acid was approximately $7.0 \mathrm{mg} / \mathrm{dL}$ irrespective of CKD stage, except for in the case of CKD $5 \mathrm{D}(8.2 \mathrm{mg} / \mathrm{dL}$ for gout and $7.6 \mathrm{mg} /$ 
$\mathrm{dL}$ for CVE). We did not separate the patients' conditions according to CKD stage due to the fear of a low response rate related to difficult questions. Further surveillance or cohort studies to deduce the target levels of serum uric acid according to CKD stage and the purpose of treatment are required.

Fear of adverse reactions and the lack of evidence are important hurdles to prescribing ULAs. In addition, the dosage of ULAs was inversely associated with the eGFR.

As mentioned above, several studies have reported an association between hyperuricemia and CKD [7-9]. In addition, there have been many clinical studies on serum uric acid reduction and its effect on renal function. However, most of them were limited by a small size and short follow-up duration. In addition, the results regarding renal function and proteinuria were inconsistent [6]. Therefore, there has been no strong recommendation made for treating AHU, even in CKD patients. However, there have been many clinical studies published after the official recommendations from Japan, the US, and Europe suggesting an effect of allopurinol on endothelial dysfunction improvement, GFR preservation, improvement of insulin resistance and systemic inflammation, and increased patient and graft survival in kidney recipients in patients with AHU [17-20].

In addition, several trials on the effects of febuxostat, topiroxostat, and newer ULAs on renal function and cardiovascular diseases have been published. Both febuxostat and topiroxostat delayed eGFR decline [21,22]. In addition, two recently released large clinical trials (the Febuxostat for Cerebral and Cardiorenovascular Events Prevention Study [FREED] and the Febuxostat versus Placebo Randomized Controlled Trial Regarding Reduced Renal Function in Patients with Hyperuricemia Complicated by Chronic Kidney Disease Stage 3 [FEATHER]) $[23,24]$ using febuxostat suggested a target serum uric acid level, which is beneficial in lowering mortality and renal progression and defined CKD patient groups who can benefit from ULAs. Febuxostat effectively reduced serum uric acid levels in both trials in 12 weeks and maintained decreased serum uric acid levels until the end of the respective investigations. Both composite cerebroCVEs and renal outcomes occurred less frequently in the febuxostat group in the FREED trial, and the study verified successful reduced serum uric acid level outcomes (serum uric acid between 5.0 and $6.0 \mathrm{mg} / \mathrm{dL}$ ) [23].
Patients with mild kidney injuries, i.e., those without proteinuria and serum creatinine levels less than the median level of all study participants, showed slower eGFR progression in the FEATHER trial [24].

Nephrologists manage CKD patients by using renin angiotensin aldosterone receptor blockers, statins, and other verified renoprotective medicines, but many CKD patients still progress to ESRD. Thus, another option to improve renal and patient outcomes in CKD patients is needed. Our focus has therefore expanded to uremic toxins and inflammatory mediators. Moreover, the management of serum uric acid is an attractive option based on experimental and clinical evidence. Currently, the CKD FIX (a placebo-controlled study giving allopurinol to CKD patients in stages 3 and 4 with proteinuria) [25] and PERL (a placebo-controlled study giving allopurinol to type 1 diabetic nephropathy patients with eGFRs of $40-100 \mathrm{~mL} / \mathrm{min} / 1.73 \mathrm{~m}^{2}$ ) [26] trials are actively ongoing, and we expect to confirm from these more clear evidence regarding the relationship between lowering serum uric acid level and renal and patient outcomes for CKD patients with a variety of conditions.

Although decreased renal function can increase the serum uric acid level and a cohort or a prospective study to find the causal relationship between AHU and patient outcomes in Korean CKD patients is needed, we think that the systemic impact of hyperuricemia does not differ with the presence of CKD. Therefore, we carefully suggest adopting the definition of hyperuricemia as a serum uric acid level of greater than $7.0 \mathrm{mg} / \mathrm{dL}$ for the general population, irrespective of CKD stage. In addition, we suggest prescribing ULAs if the serum uric acid level exceeds 8.0 $\mathrm{mg} / \mathrm{dL}$ with the consideration of tissue solubility of uric acid and targeting the serum uric acid level to less than $6.0 \mathrm{mg} / \mathrm{dL}$, as is the guideline for gout patients. However, more research is needed to establish a conclusion regarding the management of AHU in CKD patients.

This survey has a limitation, in that only $12.6 \%$ of members responded to the questionnaire. Therefore, the findings of this report may not represent the real practice patterns of nephrologists who care for CKD patients in Korea.

In conclusion, a number of registered members in the KSN caring for CKD patients treat AHU to prevent CKD progression or cerebro-cardiovascular complications mainly by using febuxostat and allopurinol. We must es- 
tablish and share Korean guidelines for the management of AHU in CKD patients.

\section{Conflicts of interest}

All authors have no conflicts of interest to declare.

\section{Authors' contributions}

Ran-hui Cha, Chul Woo Yang, and Duk-Hee Kang participated in conception or design. Ran-hui Cha participated in analysis and interpretation of data, and drafting the article or revising. Su Hyun Kim, Eun Hui Bae, Mina Yu, Beom Soon Choi, Hoon Young Choi, Sun Woo Kang, Jungho Shin, and Sang Youb Han participated in providing intellectual content of critical importance to the work. All authors read and approved of the final version to be published.

\section{References}

[1] Krishnan E. Reduced glomerular function and prevalence of gout: NHANES 2009-10. PLoS One 2012;7:e50046.

[2] Perez-Ruiz F, Dalbeth N, Bardin T. A review of uric acid, crystal deposition disease, and gout. Adv Ther 2015;32:3141.

[3] Lee JH, Choi HJ, Lee BH, et al. Prevalence of hypouricaemia and SLC22A12 mutations in healthy Korean subjects. Nephrology (Carlton) 2008;13:661-666.

[4] Ryu S, Chang Y, Zhang Y, et al. A cohort study of hyperuricemia in middle-aged South Korean men. Am J Epidemiol 2012;175:133-143.

[5] Kim Y, Kang J, Kim GT. Prevalence of hyperuricemia and its associated factors in the general Korean population: an analysis of a population-based nationally representative sample. Clin Rheumatol 2018;37:2529-2538.

[6] Ramirez MEG, Bargman JM. Treatment of asymptomatic hyperuricemia in chronic kidney disease: a new target in an old enemy - a review. JAdv Res 2017;8:551-554.

[7] Kawashima M, Wada K, Ohta H, Terawaki H, Aizawa Y. Association between asymptomatic hyperuricemia and newonset chronic kidney disease in Japanese male workers: a long-term retrospective cohort study. BMC Nephrol 2011; $12: 31$.

[8] Akasaka H, Yoshida H, Takizawa H, et al.; BOREAS-CKD1 Investigators. The impact of elevation of serum uric acid level on the natural history of glomerular filtration rate (GFR) and its sex difference. Nephrol Dial Transplant 2014; 29:1932-1939.

[9] Li L, Yang C, Zhao Y, Zeng X, Liu F, Fu P. Is hyperuricemia an independent risk factor for new-onset chronic kidney disease?: a systematic review and meta-analysis based on observational cohort studies. BMC Nephrol 2014;15:122.

[10] Kuwabara M, Niwa K, Hisatome I, et al. Asymptomatic hyperuricemia without comorbidities predicts cardiometabolic diseases: five-year Japanese cohort study. Hypertension 2017;69:1036-1044.

[11] Srivastava A, Kaze AD, McMullan CJ, Isakova T, Waikar SS. Uric acid and the risks of kidney failure and death in individuals with CKD. Am J Kidney Dis 2018;71:362-370.

[12] Yamanaka H, Metabolism TG. Essence of the revised guideline for the management of hyperuricemia and gout. Japan Med Assoc J 2012;55:324-329.

[13] Eleftheriadis T, Golphinopoulos S, Pissas G, Stefanidis I. Asymptomatic hyperuricemia and chronic kidney disease: narrative review of a treatment controversial. J Adv Res 2017;8:555-560.

[14] Song JS. Recent advances in management of gout. J Korean Med Assoc 2016;59:379-384.

[15] Nakaya I, Namikoshi T, Tsuruta Y, et al.; School of Designing Clinical Study for Nephrologists and Dialysis Physicians. Management of asymptomatic hyperuricaemia in patients with chronic kidney disease by Japanese nephrologists: a questionnaire survey. Nephrology (Carlton) 2011; 16:518-521.

[16] Schlesinger N, Moore DF, Sun JD, Schumacher HR Jr. A survey of current evaluation and treatment of gout. $J$ Rheumatol 2006;33:2050-2052.

[17] Kanbay M, Huddam B, Azak A, et al. A randomized study of allopurinol on endothelial function and estimated glomular filtration rate in asymptomatic hyperuricemic subjects with normal renal function. Clin J Am Soc Nephrol 2011;6: 1887-1894.

[18] Osadchuk L, Bashir MH, Tangirala B, et al. Effect of allopurinol on slowing allograft functional decline in kidney transplant recipients. Exp Clin Transplant 2014;12:190-194.

[19] Takir M, Kostek O, Ozkok A, et al. Lowering uric acid with allopurinol improves insulin resistance and systemic inflammation in asymptomatic hyperuricemia. J Investig Med 2015;63:924-929.

[20] Pagonas N, Kor S, Seibert FS, et al. Effects of treatment of asymptomatic hyperuricemia on graft survival and mor- 
tality in kidney transplant recipients. Ann Transplant 2016;21:350-359.

[21] Sircar D, Chatterjee S, Waikhom R, et al. Efficacy of febuxostat for slowing the GFR decline in patients with CKD and asymptomatic hyperuricemia: a 6-month, double-blind, randomized, placebo-controlled trial. Am J Kidney Dis 2015;66:945-950.

[22] Wada T, Hosoya T, Honda D, et al. Uric acid-lowering and renoprotective effects of topiroxostat, a selective xanthine oxidoreductase inhibitor, in patients with diabetic nephropathy and hyperuricemia: a randomized, doubleblind, placebo-controlled, parallel-group study (UPWARD study). Clin Exp Nephrol 2018;22:860-870.

[23] Kojima S, Matsui K, Hiramitsu S, et al; Febuxostat for Cerebral and CaRdiorenovascular Events PrEvEntion StuDy
(FREED) investigators. Febuxostat for Cerebral and CaRdiorenovascular Events PrEvEntion StuDy. Eur Heart $J$ 2019;40:1778-1786.

[24] Kimura K, Hosoya T, Uchida S, et al. Febuxostat therapy for patients with stage $3 \mathrm{CKD}$ and asymptomatic hyperuricemia: a randomized trial. Am J Kidney Dis 2018;72:798-810.

[25] Australian New Zealand Clinical Trials Registry [Internet]. Camperdown (Australia): Australian New Zealand Clinical Trials Registry, 2018 [updated 2018 Nov 30; cited 2019 Feb 11]. Available from: http://www.anzctr.org.au/Trial/Registration/TrialReview.aspx?id=343216.

[26] Maahs DM, Caramori L, Cherney DZ, et al.; PERL Consortium. Uric acid lowering to prevent kidney function loss in diabetes: the preventing early renal function loss (PERL) allopurinol study. Curr Diab Rep 2013;13:550-559. 